\title{
Improvement of Adaptive Target Tracking Protocol (ATTP) in Wireless Sensor Networks
}

\author{
Elham Ahmadi \\ Computer Engineering Department \\ Islamic Azad University,South Tehran Branch, \\ Tehran, Iran
}

\author{
Masoud Sabaei \\ Computer Engineering Department \\ Amirkabir University \\ Tehran, Iran
}

\begin{abstract}
In Adaptive Target Tracking Protocol (ATTP), at first, the total area that the target would appear there is estimated, after that some candidate wakening up areas and volunteer sampling time intervals are defined. Then by using an extended Kalman filter (EKF)-based estimation technique and energy consumption model, the best candidate wakening up area and sampling time intervals are determined. In this paper, we focus more on the definition of candidate wakening up areas. In other words, we improve the ATTP protocol in a way that the candidate wakening up areas are defined according to target's mobility model. The simulations illustrate that the improved protocol makes much progress in the accuracy of tracking.
\end{abstract}

\section{Keywords}

Target tracking; mobility model; tracking error; energy consumption; wireless sensor networks.

\section{INTRODUCTION}

Wireless Sensor Networks (WSNs) have many promising applications including environmental monitoring, traffic monitoring, fire alarming, logistics, and military sensing and tracking, and so on [1].

Inwireless sensor networks some factors are significantly effective in the accuracy of tracking: Firstly, the accurate prediction of target's path and consequently, the size and the shape of a collection of sensors, which are involved in tracking operation. The sampling time interval is also another effective factor in this issue. In other words, when we use more sensor nodes for tracking a target and smaller time intervals, we can do tracking operation with a better accuracy; but on the other hand, this kind of tracking would need more energy consumption. In a word, we need to set a trade-off between the accuracy and energy consumption during the target tracking in WSNs in order to increase the network's lifetime.

Until now a lot of researches on adaptive target tracking protocols have been done. In paper [2] a new method that is called Target Tracking Based on Mobility Model $\left(\mathrm{T}^{2} \mathrm{BM}^{2}\right)$ has been proposed, which uses Semi-Dynamic Clustering structure. The main idea of this method is that, when the target moves in different directions, to increase the accuracy of tracking it is better to use bigger size of clusters. On the other hand, when the target's motion is predictable, in order to decrease the energy consumption it is better to decrease the size of clusters. In Semi-Dynamic Clustering structure, at first, the network backbone is formed from small static clusters then during the tracking period based on the number of nodes needed to participate in tracking operation, some of these backbone clusters are merged and form a bigger cluster. In paper [3] a new classification algorithm has been introduced that by using easy classification based on velocity estimate provides a way to reduce the uncertainly in movement of target and thus the error on the estimate of target's position is reduced. In [4], a protocol forPrediction Accuracy-based Tracking Energy Saving (PATES) is well developed to conserve energy of WSNs. In [5], an energy efficient adaptive tracking algorithm called Predict-and-Mesh $(\mathrm{PaM})$ is proposed, which consists of two prediction models and a failure recovery process.

In paper [6], Adaptive Target Tracking Protocol (ATTP) has been proposed.In this protocolat first, the total area that the target would appear there is estimated, after that some candidate wakening up areas and volunteer sampling time intervals are defined. Then by using an extended Kalman filter (EKF)-based estimation technique and energy consumptionmodel the best candidate wakening up area and sampling time interval are determined. Now in this paper, we focus more on the definition of candidate wakening up areas. In other words, we improve the ATTP protocol in a way that the candidate wakening up areas are defined based on target mobility model.

The rest of this paper is organized as follows. In section 2, a brief review on ATTP protocol will be presented. In section 3, the relation between the target mobility and the shape of wakening up areas is discussed. In section 4 , the improvement on the ATTP protocol will be discussed.Also simulation results are given in section 5. Finally, conclusion and future work are introduced in Section 6.

\section{A Brief Review on ATTP}

In ATTP protocol, the network's structure is based on SDCstructure. In this structure, at first the network backbone is defined in static clustering form and then according to the number of nodes needed for tracking of a target some of these backbone clusters are merged and form the bigger cluster.

In ATTP protocol for tracking of a target at first, the whole of area which the target would probably appear there is estimated. This area is denoted as $\operatorname{Area}_{\text {Total }}(t+1)$. In mathematical approach, this area can be described as:

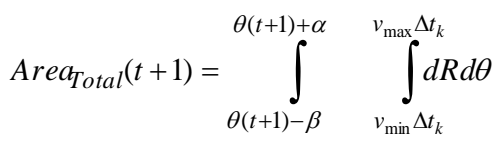

Where

- $\mathrm{V}_{\max }$ : the target's maximum velocity

- $\mathrm{V}_{\min }$ : the target's minimum velocity

- $\Delta \mathrm{t}_{\mathrm{k}}$ : the $k t h$ sampling time interval $\left(\Delta t_{k}=t_{k+1}-t_{k}\right)$

- $\theta(\mathrm{t}+1)$ : the predicted direction for target's mobility 
- $\alpha$ : the maximum angle which the target would bend toward up

- $\beta$ : the maximum angle which the target would bend toward down

It is important to note that, always $\operatorname{Area}_{\text {Total }}(t+1)$ is not considered as the wakening up area in the next tracking step. In other words, respect to energy consumption, it is better to select a smaller area as wakening up area. So in the next step, Area $_{\text {Total }}(t+1)$ is divided into several candidates wakening up areas, which are denoted as $\left\{\text { Area }_{i}\right\}_{1}^{N}$. (Figure1 shows a whole wakening up area $\mathrm{ABCD}$, which is divided into four candidate wakening up areas). After that, several candidate sampling time intervals are also considered. Then, two helpful tools are used. First, an extended Kalman filter (EKF)-based estimation technique to predict the tracking error in the next tracking step and an energy consumption model is also used to estimate the amount of energy consumption based on different candidate wakening up areas and different sampling time intervals in the next tracking step. After predicting the tracking error and energy consumption for different candidate wakening up areas and different sampling time intervals, the optimum sampling time interval and volunteer wakening up area for the next $\mathrm{T}$ secondsare selected as:

$$
\begin{array}{r}
\left(\text { Area }_{\mathrm{j}}{ }^{*}, \Delta \mathrm{t}_{\mathrm{k}} *\right)=\underset{\mathrm{j} \in \mathrm{A}, \Delta \mathrm{t}_{\mathrm{k}} \in\left[\mathrm{T}_{\text {min }}, \mathrm{T}_{\text {max }}\right]}{\operatorname{argmin}}\left\{\text { Cost_F}_{\mathrm{k}}\right]
\end{array}
$$

with

$$
C_{-} t_{-} F\left(\text { Area }_{j}, \Delta t_{k}\right)=w \frac{\Phi_{j, \Delta t_{k}}^{T}}{\Phi_{0}}+(1-w) \frac{E_{j, \Delta t}^{T}}{E_{0}}
$$

Where $A$ is the set of candidate wakening up areas, Cost_F $\left(\right.$ Area $\left._{j}, \Delta t_{k}\right)$ is the object function, if $A r e a_{j}$ is selected with the sampling time interval $\Delta \mathrm{t}_{\mathrm{k}}$. Also $\frac{\Phi_{j, \Delta t_{k}}^{T}}{\Phi_{0}}$ is the normalized tracking error and $\frac{E_{j, \Delta t}^{T} k}{E_{0}}$ is the normalized total energy consumption over $T$ seconds. And $w \in[0,1]$ is a weighting parameter used to balance the tracking accuracy and the energy consumption [6].
A
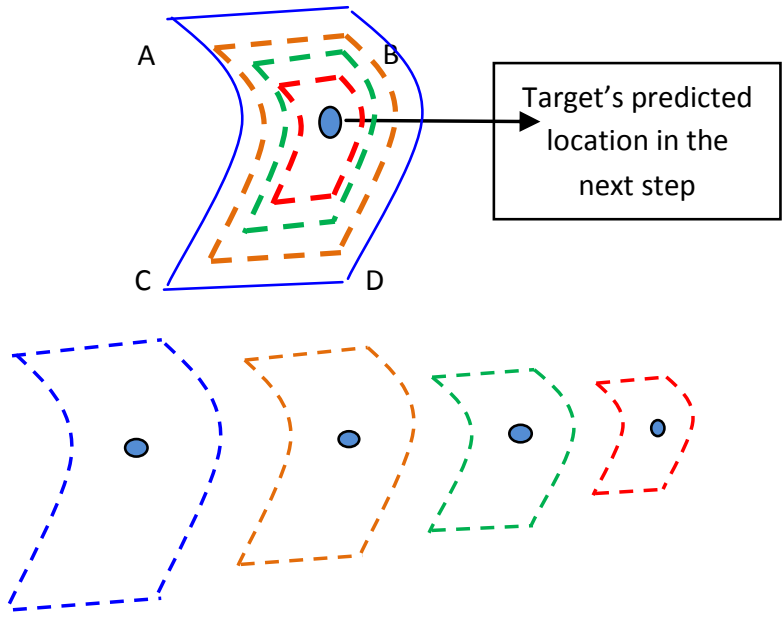

Figure 1: a whole wakening up area and four candidate wakening up areas

\section{THE EFFECT OF}

TARGET'SMOBILITY MODEL ON THE SHAPE OF WAKENING UP AREAS

In this section, we explore the relation between the target'smobility model and the shape of wakening up areas. Let's consider two scenarios. In the first scenario a person is walking, and in the second, a target like a motorcycle moves with a high velocity. In the first scenario, at every moment the target can turn right or left easily because he doesn't have inertia in his movement. So, for tracking of a target which moves in a low speed, we determine the shape of wakening up areas in the form of a circle (Figure2.a). On the other hand, for tracking a fast target such as a motorcycle, which needs more time than a walking person to turn right or left without problems, the shape of wakening up areas are defined in the form of an ellipse. Figure 2.b shows a scenario of this kind of target tracking [3].

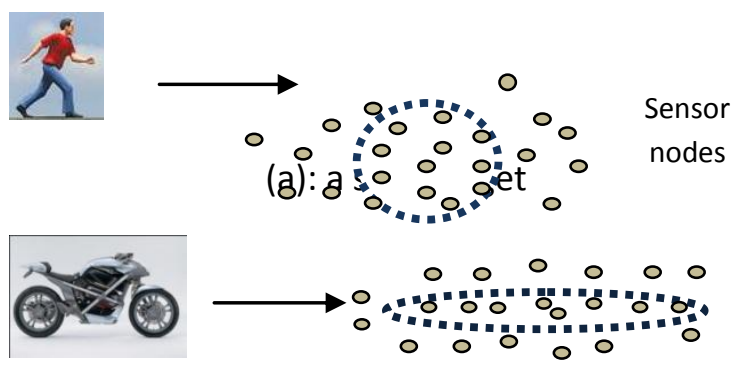

(b): a fast target

Figure 2: the shape of waking up area for tracking a slow and fast target 


\section{ATTP_AC PROTOCOL}

In this section, ATTP_AC protocol (Adaptive Target Tracking Protocol based on Adaptive Candidate wakening up areas) will be discussed. This protocol focus on this issue that how we can divide the whole of wakening up area $\left(\operatorname{Area}_{\text {Total }}(t+1)\right)$ into several candidate wakening up areas? In other words, how the candidate wakening up areas, $\left\{\text { Area }_{i}\right\}_{1}^{N}$ are defined according to target's mobility parameters. Basically, a target according to its mobility model can be placed in one of these four groups.

Group 1: Targets which are moving in fix direction but with various speeds.

Group 2:Targets which have uniform speed and move in different directions

Group 3: Targets which move with uniform speed and direction

Group 4: Targets which move with various speeds and directions

Based on the presented concepts in section 3, for tracking a target in group 1, which moves in a fix direction it is better to divide the whole of wakening up area to a series of discrete areas in a way that have maximum length. Secondly, for tracking a target in group 2, which moves in different directions during its mobility, it is better to divide the whole of wakening up area into a series of discrete areas in a way that have maximum width. Thirdly, for tracking a target in group 3 or in group 4, we divide the length and width of total wakening up area uniformly. Fig. 3 presents these concepts better. Let's suppose that Fig 3.a is a whole of wakening up area, so different type of candidate waking up areas are defined for different type of targets according to Fig 3.b,3.c and 3.d. In mathematical view, according to type of target's mobilitymodel the $i$-thcandidate area is defined according to table 1 .

Table 1: The definition of $i$-th candidate wakening up area

\begin{tabular}{|c|c|}
\hline group & The definition of $i$-th candidate wakening up area \\
\hline 1 & $\operatorname{Area}_{i}=\int_{\theta(t+1)-\frac{i \beta}{n}}^{\theta(t+1)+\frac{i \alpha}{n}} \int_{\left[v_{p r e}-v_{\min }\right] \Delta t}^{\left[v_{p r e}+v_{\max }\right] \Delta t} d R d \theta$ \\
\hline 2 & Area $_{i}=\int_{\theta(t+1)-\beta}^{\theta(t+1)+\alpha} \int_{\left[v_{p r e}-\frac{i\left(v_{p r e}-v_{\min }\right)}{n}\right] \Delta t}^{\left[v_{\text {pre }}+\frac{i\left(v_{\max }-v_{p r e}\right)}{n}\right] \Delta t}$ \\
\hline 3 or 4 & Area $_{i}=\int_{\theta(t+1)-\frac{i \beta}{n}}^{\theta(t+1)+\frac{i \alpha}{n}} \quad \int_{\left(v_{p r e}-\frac{i\left(v_{\text {pre }}-v_{\min }\right)}{n} \Delta t\right)}^{\left(v_{\text {pre }}+\frac{i\left(v_{\max }-v_{\text {pre }}\right)}{n} \Delta t\right)}$ \\
\hline
\end{tabular}

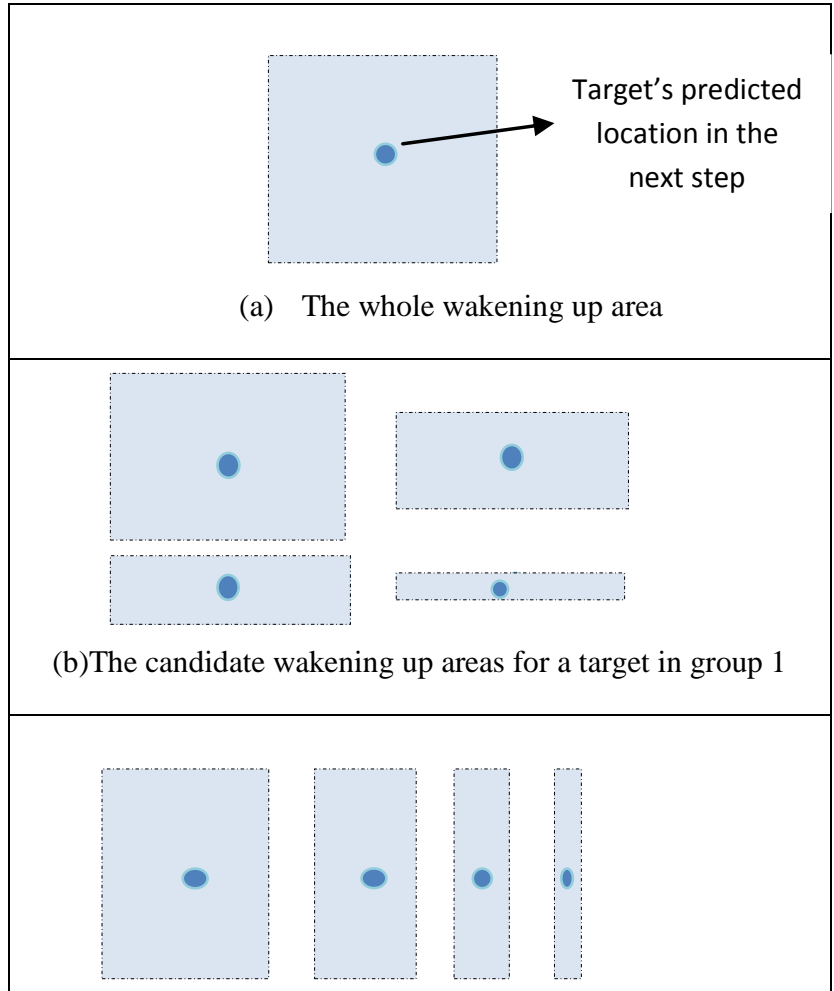

(c)The candidate wakening up areas for a target in group 2

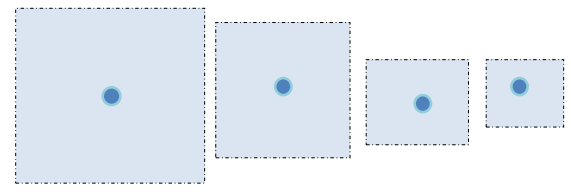

(d)The candidate wakening up areas for a target in group 3 or

Figure 3: different candidate waking up areas for different type of targets

\section{PERFORMANCE EVALUATION OF THE PROPOSED ALGORITHM}

We have used simulations to compare the accuracy of the tracking. The velocity of target is modeled by the composition of Normal distribution process and Gauss-Markov process as: $v_{n}=\lambda_{v}\left(N\left(\mu_{v}, \sigma_{v}\right)\right)+\left(1-\lambda_{v}\right)\left(\alpha v_{n-1}+(1-\alpha) \mu_{v}\right)$ Where $v_{\eta}=v(n \Delta t)$ is the $n t h$ sample of target velocity, $\mu_{v}$ is the mean value of $\mathrm{v}$, and $\sigma_{v}$ is the variance of $v$. The same equation holds for the target direction $\theta$, with corresponding values of $\mu_{\theta}$ and $\sigma_{\theta}$ as equation (7).

$\theta_{n}=\lambda_{\theta}\left(N\left(\mu_{\theta}, \sigma_{\theta}\right)\right)+\left(1-\lambda_{\theta}\right)\left(\alpha \theta_{n-1}+(1-\alpha) \mu_{\theta}\right)$

By coefficient $\lambda_{v}$ and $\lambda_{\theta}$, we can control target's mobility model in simulations. In other words, $\lambda_{v}=1$ means that the target'sspeed changes randomly.As well as, $\lambda_{v}=0$ means that, the target is moving in uniform speed. On the other hand, $\lambda_{\theta}=1$ means that the target is moving in different directions 
and $\lambda_{\theta}=0$ indicates that the target moves in a uniform direction.

The sensor network field is considered as a rectangular area of $200 \times 200 \mathrm{~m}^{2}$, where 1,000 sensor nodes have been deployed in grid structure. All nodes are identical and so they have the same initial resources.

We evaluated our proposed method, ATTP-AC protocol (Adaptive Target Tracking Protocol based on Adaptive Candidate wakening up areas) by tracking error with ATTP which has been proposed in paper [6]. In the simulations, in order to implement different mobility models, we increase the coefficients $\lambda_{v}$ and $\lambda_{\theta}$ continuously in relations (7) and

(8).Furthermore, we suppose that the number of sensor nodes which participate in each stage of tracking is constant. As we see in Figure 4 and Figure5, when the coefficient $\lambda_{v}$ and $\lambda_{\theta}$ increases continuously, consequently the tracking error increases but the rate of increasing error in ATTP_AC is less than ATTP, because in ATTP-AC protocol, we have selected nodes to be waked up in the next tracking step based on target's mobility model.

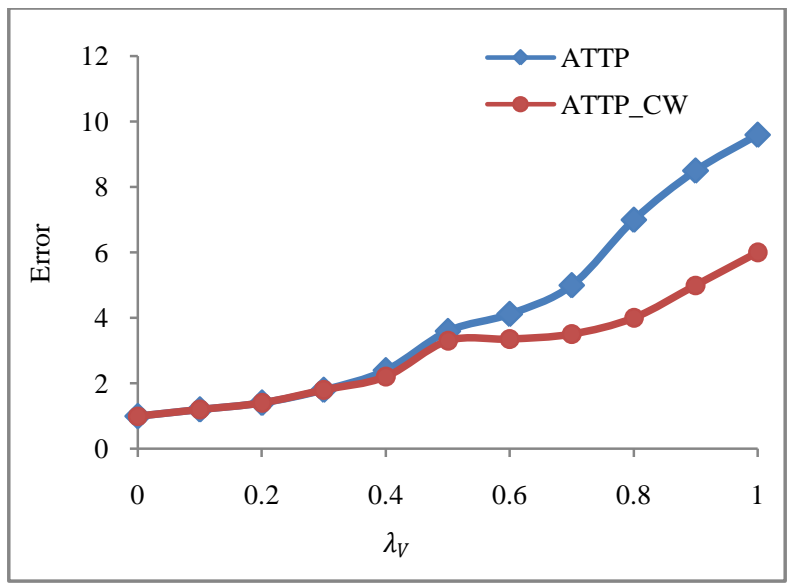

Figure 4: tracking error in different mobility models based on $\lambda_{v}$

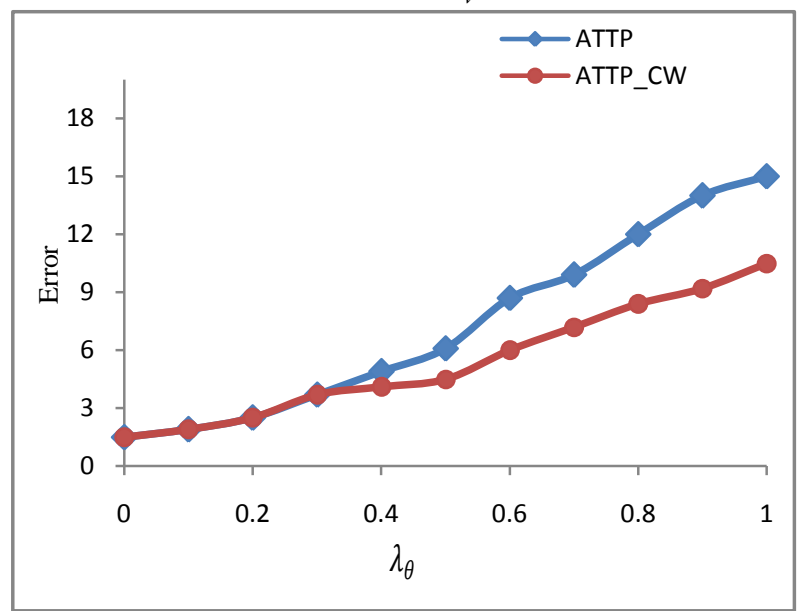

Figure 5: tracking error in different mobility models based on $\lambda_{\theta}$

\section{CONCLUSION}

This paper has improved adaptive target tracking protocol in paper [6]. In the proposed method, the candidate wakening up areasare selected according to target's mobility model. In other words, for tracking a target which moves in a fix direction, the whole of wakening up area is divided to a series of discrete areas in a way that have maximum length. Secondly, for tracking a target which moves in different directions during its mobility, the whole of wakening up area is divided into a series of discrete areas in a way that have maximum width. Thirdly, for tracking a target in group 3 or in group 4, we divide the length and width of total wakening up area uniformly. It is proposed toward another possible area of work to estimate the candidate wakening up areas more accurately based on more technical analysis of target mobility.

\section{REFERENCES}

[1] H. W. Tsai, C. P. Chu, T. S. Chen, "Mobile object tracking in wireless sensor networks," Computer Communication, 2007, 30(8): 1811-1825.

[2] E. Ahmadi, M. Sabaei, M. H. Ahmadi "A New AdaptiveMethod for Target Tracking in Wireless Sensor Networks",International Journal of Computer Applications, volume22,No.9, 2011, pp.21-29.

[3] Sara Pino-Povedano, Francisco-Javier Gonzalez Serrano, "Distributed Tracking and Classification of Targets with Sensor Networks," IEEE 2009.

[4] Z. Guo, M. Zhou and L. Zakrevski, "Optimal Tracking Interval for predictive Tracking in wireless Sensor Networks," IEEE Communication Letters, vol. 9, No.9, Sept 2005.

[5] L. Yang, C. FENG, J. W. Rozenblit , and H. Qiao, "Adaptive Tracking in distributed Wireless Sensor Networks," in proc. Of $13^{\text {th }}$ Annual IEEE International Symposium and Workshop on Engineering of Computer Based Systems, pp. 9, Mar. 2006.

[6] E. Ahmadi, M. Sabaei, " A New Adaptive Target Tracking Protocol in Wireless Sensor Networks", International Journal of Computer Applications, volume28, No.10, 2011, pp.5-11. 